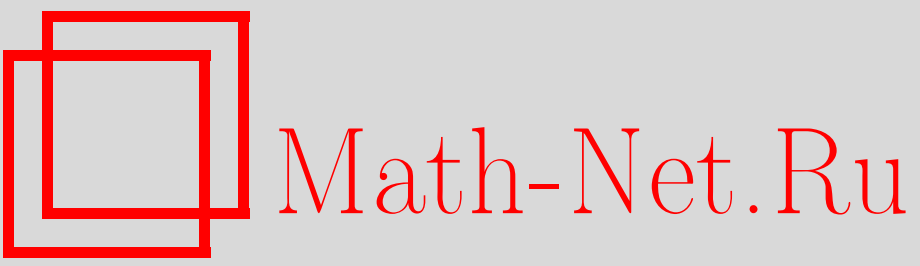

С. П. Царев, Факторизация линейных дифференциальных операторов с частными производными и метод Дарбу интегрирования нелинейных уравнений с частными производными, ТМ Ф, 2000, том 122, номер 1, 144-160

DOI: https://doi.org/10.4213/tmf561

Использование Общероссийского математического портала Math-Net.Ru подразумевает, что вы прочитали и согласны с пользовательским соглашением

http: //www . mathnet.ru/rus/agreement

Параметры загрузки:

IP: 54.210 .77 .194

26 апреля 2023 г., 14:34:56 


\section{ФАКТОРИЗАЦИЯ ЛИНЕЙНЫХ ДИФФЕРЕНЦИАЛЬНЫХ ОПЕРАТОРОВ С ЧАСТНЫМИ ПРОИЗВОДНЫМИ И МЕТОД ДАРБУ ИНТЕГРИРОВАНИЯ НЕЛИНЕЙНЫХ УРАВНЕНИЙ С ЧАСТНЫМИ ПРОИЗВОДНЫМИ}

На основе нового определения обобщенной факторизации линейных операторов с частными производными обсуждаются возможные обобщения понятия интегрируемости по Дарбу нелинейных диффференциальных уравнений с частными производными.

\section{1. ВВЕДЕНИЕ}

B XIX веке развитие различных методов нахождения точных решений нелинейных дифференциальных уравнений с частными производными привело к созданию методов Лагранжа, Монжа, Буля и Ампера. Дарбу [1] обобщил метод Монжа (известный как метод промежуточных интегралов), предложив наиболее мошный из имеющихся на конец прошлого века методов интегрирования.

Недавно в серии работ [2-4] метод Дарбу был переосмыслен и представлен в более точной и эффективной (хотя и не вполне алгоритмичной) форме. Для случая одного нелинейного уравнения с частными производными относительно одной функции от двух независимых переменных вида

$$
u_{x y}=f\left(x, y, u, u_{x}, u_{y}\right)
$$

идея указанных работ состояла в линеаризации уравнения (1). Используя подстановку $u(x, y) \rightarrow u(x, y)+\epsilon v(x, y)$ и пренебрегая слагаемыми с $\epsilon^{n}, n>1$, мы получаем линейное уравнение в частных производных (ЛУЧП)

$$
v_{x y}=A v_{x}+B v_{y}+C v
$$

\footnotetext{
*Красноярский государственный педагогический университет, Красноярск, Россия. E-mail: tsarev@edk.krasnoyarsk.su
} 
с коэффициентами $A, B, C$, зависяшими от $x, y, u, u_{x}, u_{y}$. Уравнения типа (2) изучались еше Лапласом, который создал метод интегрирования на основе их преобразования (называемый иногда каскадным методом Лапласа) [5]. Прежде всего отметим, что для соответствующего линейного дифференциального оператора с частными производными (ЛДОЧП) выполнены следующие равенства:

$$
L=D_{x} \circ D_{y}-A \cdot D_{x}-B \cdot D_{y}-C=\left(D_{x}-B\right) \circ\left(D_{y}-A\right)+H=\left(D_{y}-A\right) \circ\left(D_{x}-B\right)+K,
$$

где $D_{x}=\partial / \partial x, D_{y}=\partial / \partial y$. Величины $H=D_{x} A-A B-C, K=D_{y} B-A B-C$ называются инвариантами Лапласа уравнения (2). Поэтому, если $H \equiv 0$ либо $K \equiv 0$, указанный оператор второго порядка $L$ факторизуется в "обычном" смысле и решения уравнения (2) могут быть найдены посредством квадратуры. Если оба инварианта $H$ и $K$ обрашаются в нуль, то $L$ является левым наименьшим общим кратным (ILCM) двух Л ДОЧП первого порядка. Если оба $H, K$ не равны нулю, то можно применить к $L$ одно из двух преобразований Лапласа: $L \rightarrow L_{1}, L \rightarrow L_{-1}$, используя подстановки

$$
v_{1}=\left(D_{y}-A\right) v, \quad v_{-1}=\left(D_{x}-B\right) v .
$$

Эти обратимые преобразования приводят к двум другим ЛДОЧП второго порядка $L_{1}$, $L_{-1}$ той же формы (3) с другими коэффициентами, если и только если $H \neq 0$ (соответственно $K \neq 0$ ). В случае общего положения получаем две бесконечные последовательности

$$
\begin{aligned}
& L \rightarrow L_{1} \rightarrow L_{2} \rightarrow \cdots, \\
& L \rightarrow L_{-1} \rightarrow L_{-2} \rightarrow \cdots .
\end{aligned}
$$

Если какая-то их этих последовательностей конечна (т.е. соответствующий инвариант Лапласа обрашается в нуль на некотором шаге и преобразование Лапласа не может быть далее применено), конечный ЛДОЧП $L_{i}$ тривиально факторизуем, и, следовательно, применяя обратные преобразования, мы можем в конечной форме (в квадратурах) проинтегрировать исходное уравнение.

Разумеется, в нашем случае мы должны принимать во внимание исходное уравнение (1), выполняя все вычисления инвариантов Лапласа и преобразований Лапласа (что позволяет нам выразить все смешанные производные $u$ через $x, y, u$ и несмешанные производные $\left.u_{x \cdots x}, u_{y} \cdots y\right)$.

Tеорема 1 ([2-4]). Скалярное гиперболическое уравнение (1) интегрируемо по Дарбу, если и только если обе последовательности преобразований Лапласа конечныр.

В работах $[2,3]$ этот метод был обобщен на произвольные гиперболические уравнения второго порядка с двумя независимыми переменными $F(x, y, u$, $\left.u_{x}, u_{y}, u_{x x}, u_{x y}, u_{y y}\right)=0$. 
Метод Дарбу (как утверждал Дарбу в [1]) в приншипе "применим к уравнениям любого порядка с произвольным количеством независимых переменных и даже к системам таких уравнений"; однако в [1] и последующих многочисленных работах Гурса, Го, Госса, Вессио и др. конкретные результаты были получены лишь для одного уравнения второго порядка с одной независимой и двумя зависимыми переменными.

В данной статье мы показываем, что интегрируемость по Дарбу тесным образом связана с (обобщенной) факторизуемостью соответствующего линеаризованного оператора. Прежде всего выясним, что можно сказать о факторизуемости оператора $L$ (3). Как мы отмечали, преобразование Лапласа разрушает тривиальную факторизуемость $L=\left(D_{x}-B\right) \circ\left(D_{y}-A\right)$ и соответственно $L=\left(D_{y}-A\right) \circ\left(D_{x}-B\right)$. Мы даем ниже подходяшую модификацию определения факторизуемости. В смысле этого определения в разделе 6 будут доказаны следуюшие теоремы.

Teopema 2. Onepamop $L=D_{x} \circ D_{y}-a(x, y) D_{x}-b(x, y) D_{y}-c(x, y)$ uмеет нетривиальный правый обобщенный дивизорный идеал, если и только если одна из последовательностей Лапласа конечна.

Teopema 3. Onepamop $L=D_{x} \circ D_{y}-a(x, y) D_{x}-b(x, y) D_{y}-c(x, y)$ есть наименьшее левое кратное двух обобщенных правых дивизорных идеалов, если и только если обе последовательности Лапласа конечны.

Доказательства этих теорем конструктивны и остаются справедливыми для произвольного дифференциального поля коэффициентов, поэтому они могут быть применены к уравнению (2).

Наше определение обобшенной факторизашии также может обеспечить обобшение понятия интегрируемости по Дарбу на случай (скалярного) нелинейного дифференциального уравнения с частными производными с произвольным числом независимых переменных (см. ниже раздел 7$)$.

\section{2. ФАКТОРИЗАЦИЯ ЛИНЕЙНЫХ ДИФФЕРЕНЦИАЛЬНЫХ ОПЕРАТОРОВ}

Факторизация (разложение на множители) часто используется для упрощения процедур решения полиномиальных уравнений. Естественные аналоги основных результатов теории факторизации многочленов одной переменной сушествуют также для линейных обыкновенных дифференциальных операторов (ЛОДО)

$$
L=f_{0}(x) D^{n}+f_{1}(x) D^{n-1}+\cdots+f_{n}(x), \quad D=\frac{d}{d x}, \quad \operatorname{ord}(L)=n .
$$

Их коэффициенты $f_{s}(x)$ считаются принадлежашими некоторому дифференциальному полю K. Для простоты можно предполагать, что это некоторое поле функций, которое замкнуто относительно операции дифференцирования. В большинстве приложений $\mathbf{K}=\mathbf{Q}(x)$-поле рациональных функций с рациональными коэффициентами. Например, широко известно, что любой полином разлагается в произведение неприводимых (над 
данным полем коэффициентов) полиномов единственным образом. Для ЛОДО аналогичный результат был доказан Ландау [6] и в более точной форме Леви: любые два разложения заданного ЛОДО $L$ в произведение (композицию) неприводимых ЛОДО $L=P_{1} \circ \cdots \circ P_{k}=\bar{P}_{1} \circ \cdots \circ \bar{P}_{p}$ имеют одинаковое число множителей (факторов), т.е. $k=p$, и множители $P_{i}, \bar{P}_{j}$ имеют одинаковые порядки (как дифференциальные операторы) с точностью до перестановки. Здесь и далее мы под произведением ЛОДО понимаем их композицию как операторов. Некоммутативность такого произведения ЛОДО влечет за собой некоторые дополнительные усложнения [7].

Теория факторизации ЛОДО (теория Леви-Оре) была развита в работах [8-10]. С алгоритмической точки зрения факторизация ЛОДО впервые рассматривалась в [11], где был дан набросок алгоритма факторизации ЛОДО для простейшего (и важнейшего) случая дифференциального поля рациональных функций с алгебраическими коэффициентами (т.е. $\mathbf{K}=\overline{\mathbf{Q}}(x)$ ). В последние десять лет были предложены многочисленные уточнения, усовершенствования и альтернативные алгоритмы [7].

K сожалению, подобное тривиальное определение факторизации как представления в виде композиции линейных операторов меньших порядков не обладает хорошими свойствами для ЛДОЧП.

Следующий интересный пример принадлежит Ландау (см. [12]). Если

$$
\begin{gathered}
P=D_{x}+x D_{y}, \quad Q=D_{x}+1, \\
R=D_{x}^{2}+x D_{x} D_{y}+D_{x}+(2+x) D_{y},
\end{gathered}
$$

то $L=Q \circ Q \circ P=R \circ Q$. С другой стороны, оператор $R$ абсолютно неприводим, т.е. его невозможно разложить в композицию операторов первого порядка с коэффициентами в любом расширении $\mathbf{Q}(x, y)$, что легко проверить прямым вычислением.

Этот пример показывает, что для построения “хорошей" теории факторизации ЛДОЧП необходимо использовать некоторое обобщение понятия фактора (делителя, дивизора). Подобные обобшения обычны в коммутативной алгебре. Как один из первых примеров упомянем теорию Куммера-Дедекинда дивизоров (идеалов) в кольцах целых алгебраических чисел. Поскольку не все идеалы в этих кольцах (вообще говоря) главные, т.е. порождены одним элементом, мы получаем расширение понятия факторизации; этого оказывается достаточно (см., к примеру, [13, гл. 12]) для доказательства единственности разложения целого алгебраического числа (точнее, главного идеала, который оно порождает) из данного конечного расширения $\mathbf{Q}$ в произведение простых "идеальных" дивизоров.

Для некоммутативного случая кольца ЛОДО (с коэффициентами в некотором дифференциальном поле) легко (с помощью алгоритма Евклида деления с остатком) доказать, что любой левый или правый идеал в кольце ЛОДО $\mathbf{Q}(x)\left[D_{x}\right]$ - главный; данное кольцо не имеет нетривиальных двусторонних идеалов. Поэтому нет возможности (и необходимости) "идеального" обобшения понятия дивизора. Единственным следствием некоммутативности оказывается “сходство” факторов в различных факторизациях данного ЛОДО $[7,10])$. Другой широко известньй пример “единственности факторизации" - классическая теорема Жордана-Гельдера в теории конечных групп (или конечно порожденных модулей). 
В последующих разделах дается одно из возможных обобщений понятия факторизации для ЛДОЧП, которое позволяет объяснить пример Ландау и описать свойства факторизации операторов Лапласа (гиперболических ЛДОЧП второго порядка) в цепочке преобразований Лапласа.

\section{3. ДИВИЗОРНЫЕ ИДЕАЛЫ В КОЛЬЦЕ ЛДОЧП}

Мы будем изучать общие ЛДОЧП

$$
L=\sum_{|\vec{i}| \leqslant m} a_{i_{1} \ldots i_{n}}(\vec{x}) D_{x_{1}}^{i_{1}} D_{x_{2}}^{i_{2}} \ldots D_{x_{n}}^{i_{n}}
$$

$|\vec{i}|=i_{1}+\cdots+i_{n}, \vec{x}=\left(x_{1}, \ldots, x_{n}\right), D_{x_{i}}=\partial / \partial x_{i}, \operatorname{ord}(L):=m$. Для простоты и без потери общности можно предположить, что число независимых переменных $n=2, x:=x_{1}$, $y:=x_{2}$ и коэффициенты $a_{i j}(x, y)$ в $(6)$ являются рациональными функциями с рациональными коэффициентами, $a_{i j}(x, y) \in \mathbf{Q}(x, y), L \in \mathbf{Q}(x, y)\left[D_{x}, D_{y}\right]$. Кольцо ЛДОЧП не имеет нетривиальных двусторонних идеалов, левые (правые) идеалы более не являются главными в общем случае. Это позволяет предложить следующее определение (переформулировка обычного определения для ЛОДО в силу того, что все идеалы в кольце $\mathbf{Q}(x)\left[D_{x}\right]$ - главные): вместо факторизации

$$
L=L_{1} \circ \cdots \circ L_{k}
$$

оператора $L$ как элемента кольца мы рассмотрим возрастающие цепочки соответствующих левых (правых) главных идеалов

$$
|L\rangle \subset\left|L_{2} \circ \cdots \circ L_{k}\right\rangle \subset\left|L_{3} \circ \cdots \circ L_{k}\right\rangle \subset \cdots \subset\left|L_{k}\right\rangle \subset|1\rangle .
$$

Будем обозначать левый (правый) идеал, порожденный ЛДОЧП $L$, как $|L\rangle$ (соответственно $\langle L|)$. Неприводимость факторов соответствует максимальности такой цепочки, т.е. невозможности вставить промежуточные идеалы между какими-либо двумя элементами этой цепочки. K сожалению, это простейшее обобщение также неудачно для ЛДОЧП: обычно всегда можно вставить сколь угодно много промежуточных идеалов между некоторыми из них, поэтому длина цепочек (8) для данного ЛДОЧП может быть неограниченной. $\mathrm{K}$ примеру, для произвольного ЛОДО $L \in \mathbf{Q}(x)\left[D_{x}\right] \subset$ $\mathbf{Q}(x, y)\left[D_{x}, D_{y}\right]$ можно взять $|L\rangle \subset|L\rangle+\left|D_{y}^{m}\right\rangle \subset|L\rangle+\left|D_{y}^{m-1}\right\rangle \subset \cdots \subset|L\rangle+\left|D_{y}\right\rangle \subset$ $\mathbf{Q}(x, y)\left[D_{x}, D_{y}\right]$. Даже простейший оператор $D_{x}$ становится “приводимым"! Можно заключить, что множество всех идеалов слишком "велико". Для коммутативного случая полиномов многих переменных (где также сушествуют подобные примеры) мы можем ограничиться главными идеалами и получить конечные цепочки. Однако для ЛДОЧП множество главных идеалов слишком "мало́", как показывает пример Ландау. Другой важный для нас недостаток семейства главных идеалов кольца ЛДОЧП состоит в отсутствии наименьших общих кратных и наибольших общих делителей: для двух операторов $P, Q$ в (5) пересечение соответствуюших главных идеалов $|P\rangle \cap|Q\rangle$ (их наименьшее общее кратное, LCM) может не быть главным идеалом. Легко явно проверить, 
что нет общего левого кратного для $P, Q$ второго порядка, но имеются два линейно независимых оператора третьего порядка, деляшихся справа на $P, Q$ :

$$
\begin{aligned}
L_{31} & =\left(x D_{x} D_{y}+(x-1) D_{y}-D_{x}-1\right) \circ P= \\
& =\left(x^{2} D_{y}^{2}+x D_{x} D_{y}-(x+1) D_{y}-D_{x}\right) \circ Q, \\
L_{32} & =\left(D_{x}^{2}+2 D_{x}+1\right) \circ P=Q \circ Q \circ P= \\
& =\left(D_{x}^{2}+x D_{x} D_{y}+(x+2) D_{y}+D_{x}\right) \circ Q=R \circ Q,
\end{aligned}
$$

поэтому нет "наименьшего" левого кратного. Аналогично мы можем проверить, что эти операторы $L_{31}, L_{32}$ имеют лишь $Q, P$ в качестве своих правых обших делителей, поэтому $L_{31}, L_{32}$ не обладают “наибольшим" правым общим делителем.

Ниже мы определим "промежуточное" множество идеалов "коразмерности 1", которое шире, чем множество главных идеалов, но у́же множества всех идеалов. Как будет показано, это новое множество “дивизорных идеалов" обеспечивает необходимые свойства факторизации, аналогичные свойствам факторизации обычных коммутативных полиномов и ЛОДО. В этом и состоит наш основной результат.

Легко проверить, что для каждого конечного множества ЛДОЧП $L_{1}, \ldots, L_{k}$ можно алгоритмически найти некоторое их левое общее кратное (1CM - left Common Multiple) вплоть до заданного порядка $N$. Возьмем $M_{1} \circ L_{1}=\cdots=M_{k} \circ L_{k} \mathrm{c} \operatorname{ord}\left(M_{i}\right)=$ $N-\operatorname{ord}\left(L_{i}\right)$ и неопределенными коэффициентами. Отсюда мы получим систему линейных алгебраических (не дифференциальных) уравнений на коэффициенты $M_{i}$. Число уравнений в этой линейной системе будет меньше, чем число неизвестных коэффициентов для достаточно большого $N$, поэтому множество общих левых кратных всегда непусто.

Все эти и нижеследующие результаты, разумеется, верны и при замене левых идеалов на правые, достаточно применить обычную операцию сопряжения.

ОПРЕДЕлЕНИЕ 1 . Левый идеал $1 \mathrm{LCM}\left(\left|L_{1}\right\rangle, \ldots,\left|L_{k}\right\rangle\right):=\left|L_{1}\right\rangle \cap \cdots \cap\left|L_{k}\right\rangle$ называется левым наименьшим обшим кратным для Л ДОЧП $L_{i}$.

Этот идеал 1LCM всегда непуст и в общем случае может оказаться не главным.

ОПРЕДЕЛЕНИЕ 2. Назовем два ЛДОЧП $L, R$ (обобщенной) дивизорной парой операторов для ЛДОЧП $M$, если сушествуют такие ЛДОЧП $X, Y, Q$, что

$$
\begin{gathered}
X \circ M=Y \circ R, \\
X \circ L=Y \circ Q .
\end{gathered}
$$

Неформально можно представить себе, что $M \cong L \circ R$, и если действительно $M$ факторизуется в произведение $L$ и $R$, то, разумеется, мы можем выбрать $X=Q=1$, $Y=L$ в (9). Дивизорная пара операторов называется нетривиальной, если $\operatorname{ord}(L)>0$, $\operatorname{ord}(R)>0$ и $L, R$ не делятся на $M$, т.е. $L \neq M \circ P, R \neq K \circ M$. В таком случае будем говорить, что операторы $M$ и $R$ ( $M$ и $L$ ) имеют нетривиальный (обобщенный) правый (соответственно левый) делитель (дивизор). 
ЛЕмма 1. Если выполнено условие (9) и для некоторых ЛДОЧП $X_{1}, Y_{1}$ имеем $X_{1} \circ M=Y_{1} \circ R$, mo $X_{1} \circ L=Y_{1} \circ Q$.

ДокАЗАтЕльство. Найдем некоторое левое обшее кратное $X_{1}, X: \widetilde{X}=\bar{X} \circ X_{1}=$ $\bar{X}_{1} \circ X$. Тогда $\bar{X} \circ X_{1} \circ M=\bar{X} \circ Y_{1} \circ R=\bar{X}_{1} \circ X \circ M=\bar{X}_{1} \circ Y \circ R$ и (поскольку кольцо ЛДОЧП не имеет делителей нуля) $\bar{X} \circ Y_{1}=\bar{X}_{1} \circ Y$ и $\bar{X}_{1} \circ Y \circ Q=\bar{X}_{1} \circ X \circ L=\bar{X} \circ$ $X_{1} \circ L=\bar{X} \circ Y_{1} \circ Q$, откуда $X_{1} \circ L=Y_{1} \circ Q$.

Следовательно, $(9)$ не зависит от выбора $\operatorname{LCM}(M, R)=X \circ M=Y \circ R$.

ЛЕмма 2. Если выполнено (9) и выбрано некоторое правое общее кратное $M, L$, $M \circ \bar{X}=L \circ \bar{Y}, m o$

$$
\begin{aligned}
M \circ \bar{X} & =L \circ \bar{Y}, \\
R \circ \bar{X} & =Q \circ \bar{Y} .
\end{aligned}
$$

ДокаЗАТЕЛЬСТво. $Y \circ Q \circ \bar{Y}=X \circ L \circ \bar{Y}=X \circ M \circ \bar{X}=Y \circ R \circ \bar{X} \Rightarrow Q \circ \bar{Y}=R \circ \bar{X}$.

Из (9) и леммы 1 заключаем, что множество операторов $L$, образующих обобщенные операторные пары с фиксированными $R, M$, образуют правый идеал; из (10) видно, что для фиксированных $M, L$ операторы $R$ образуют левы й идеал.

ОпредЕлЕниЕ 3 . Левый идеал $\mid R\}$ и правый идеал $\{L \mid$ образуют (обобщенную) дивизорную пару идеалов для ЛДОЧП $M$ (мы будем обозначать этот факт следующим образом: $\{L|M| R\})$, если:

а) любые два оператора $R \in \mid R\}, L \in\{L \mid$ образуют дивизорную пару операторов для $M$, т.е. имеет место $(9)$;

б) если некоторый ЛДОЧП $L$ образует дивизорные пары операторов для $M$ с кажсдым.м $R \in \mid R\}$, то $L \in\{L \mid$;

в) если некоторый ЛДОЧП $R$ образует дивизорные пары операторов для $M$ с кажсдыıм $L \in\{L \mid$, то $R \in \mid R\}$.

Лемма 3. Пусть $\{L|M| R\}$ - дивизорная пара идеалов для $M$. Тогда для любого $\left.M_{1} \in \mid R\right\}$ можсно найти единственный правый идеал $\left\{Q_{1} \mid\right.$ такой, что $\left\{Q_{1}\left|M_{1}\right| R\right\}$.

ДокАЗАТЕЛЬСТво. Поскольку $\left.M_{1} \in \mid R\right\}$, то для всякого $L \in\{L \mid$ имеется единственный (лемма 1) $Q_{1}$ такой, что $\exists X_{1}, Y_{1}$, для которых

$$
\begin{gathered}
X_{1} \circ M=Y_{1} \circ M_{1}, \\
X_{1} \circ L=Y_{1} \circ Q_{1} .
\end{gathered}
$$

Возьмем некоторое левое обшее кратное операторов $M_{1}, R$ с $\left.R \in \mid R\right\}: Z_{1}=X_{M_{1}}$ 。 $M_{1}=Y_{M_{1}} \circ R$, и некоторое левое общее кратное операторов $Y_{1}, X_{M_{1}}: Z_{2}=\bar{Y}_{1} \circ X_{M_{1}}=$ $\bar{X}_{M_{1}} \circ Y_{1}$. Тогда $\bar{X}_{M_{1}} \circ X_{1} \circ M=\bar{X}_{M_{1}} \circ Y_{1} \circ M_{1}=\bar{Y}_{1} \circ X_{M_{1}} \circ M_{1}=\bar{Y}_{1} \circ Y_{M_{1}} \circ R$, и мы получаем $\bar{X}_{M_{1}} \circ X_{1} \circ M=\bar{Y}_{1} \circ Y_{M_{1}} \circ R$. С помощью леммы 1 можно заключить, что $\bar{X}_{M_{1}} \circ X_{1} \circ L=\bar{Y}_{1} \circ Y_{M_{1}} \circ Q$ для любого $L \in\{L \mid$ и соответствующего $Q$. Следовательно, 
$\bar{Y}_{1} \circ Y_{M_{1}} \circ Q=\bar{X}_{M_{1}} \circ X_{1} \circ L=\bar{X}_{M_{1}} \circ Y_{1} \circ Q_{1}=\bar{Y}_{1} \circ X_{M_{1}} \circ Q_{1}$. Сокрашая $\bar{Y}_{1}$, получаем $Y_{M_{1}} \circ Q=X_{M_{1}} \circ Q_{1}$, т.e.

$$
\begin{aligned}
X_{M_{1}} \circ M_{1} & =Y_{M_{1}} \circ R, \\
X_{M_{1}} \circ Q_{1} & =Y_{M_{1}} \circ Q .
\end{aligned}
$$

Равенства (12) показывают, что любое $Q_{1}$ в (11) ( $Q_{1}$ зависит от $L \in\{L \mid)$ образует дивизорную пару операторов для $M_{1}$ с любым $\left.R \in \mid R\right\}$, т.е. условие "а" определения 3 выполнено. Чтобы доказать условие "в" этого определения для $\left\{Q_{1}\left|M_{1}\right| R\right\}$, предположим, что (12) верно для некоторого Л ДОЧП $R$ и всех $Q_{1}$, полученных из (11) с $L \in\{L \mid$. Фиксируя $X_{M_{1}}, Y_{M_{1}}, X_{1}, Y_{1}$ и используя то же определение для $\bar{X}_{M_{1}}, \bar{Y}_{M_{1}}: Z_{2}=\bar{Y}_{1}$ 。

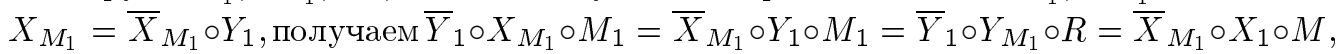
$\bar{Y}_{1} \circ X_{M_{1}} \circ Q_{1}=\bar{Y}_{1} \circ Y_{M_{1}} \circ Q=\bar{X}_{M_{1}} \circ Y_{1} \circ Q_{1}=\bar{X}_{M_{1}} \circ X_{1} \circ L$, тем самым

$$
\begin{aligned}
\bar{X}_{M_{1}} \circ X_{1} \circ M & =\bar{Y}_{1} \circ Y_{M_{1}} \circ R, \\
\bar{X}_{M_{1}} \circ X_{1} \circ L & =\bar{Y}_{1} \circ Y_{M_{1}} \circ Q,
\end{aligned}
$$

т.е. равенства (9) выполнены (лемма 1) для этого $R$ и всех $L \in\{L \mid$, поэтому $R \in \mid R\}$. Условие "б" определения 3 доказывается тем же способом: если (12) выполнено для некоторого ЛДОЧП $Q_{1}$ и всех $\left.R \in \mid R\right\} \quad\left(Q, X_{M_{1}}, Y_{M_{1}}\right.$ зависят от $\left.R\right)$, возьмем $R=$ $M \in \mid R\}$. Равенства (12) преврашаются в равенства

$$
\begin{aligned}
\widetilde{X}_{M_{1}} \circ M_{1} & =\widetilde{Y}_{M_{1}} \circ M, \\
\widetilde{X}_{M_{1}} \circ Q_{1} & =\widetilde{Y}_{M_{1}} \circ \widetilde{Q} .
\end{aligned}
$$

В этом случае, как видно из (11), можно положить (лемма 1) $\widetilde{X}_{M_{1}}=Y_{1}, \widetilde{Y}_{M_{1}}=X_{1}$, $L:=\widetilde{Q}$. Следовательно, мы имеем (11), (12) с $R \in \mid R\}$, и нам необходимо доказать (9) для построенных $L, Q, M, R$. Вновь имеем цепочку равенств $Z_{2}=\bar{Y}_{1} \circ X_{M_{1}}=\bar{X}_{M_{1}} \circ Y_{1}$, $\bar{X}_{M_{1}} \circ Y_{1} \circ M_{1}=\bar{X}_{M_{1}} \circ X_{1} \circ M=\bar{Y}_{1} \circ X_{M_{1}} \circ M_{1}=\bar{Y}_{1} \circ Y_{M_{1}} \circ R, \bar{X}_{M_{1}} \circ X_{1} \circ L=\bar{X}_{M_{1}} \circ$ $Y_{1} \circ Q_{1}=\bar{Y}_{1} \circ X_{M_{1}} \circ Q_{1}=\bar{Y}_{1} \circ Y_{M_{1}} \circ Q$, т.е. вновь получаем соотношения (13) или (9) (в силу леммы 1 ).

Лемма 3 утверж дает, что правые части в паре $\{L|M| R\}$ могут быть внутренним образом (независимо от $M$ ) охарактеризованы как некоторые специальные левые идеалы. То же верно для $\{L \mid$. Мы будем называть такие идеалы $\mid R\}$ правыми дивизорными идеалами, или r.d.i. (right divisor ideals; они являются левыми идеалами кольца ЛДОЧП), и $\{L \mid-$ левыми дивизорныцми идеалами, или 1.d.i. (left divisor ideals; они являются правы.ми идеалами кольца Л ДОЧП). Любой главный левый идеал есть r.d.i.: $\left.\left|R_{0}\right\rangle=\mid R_{0}\right\}$, поскольку с очевидностью имеем $\left\{1\left|R_{0}\right| R_{0}\right\}$, т.е. выполнено (9) с $R=P \circ R_{0}, M=R_{0}$, $Y=1, X=P, Q=P \circ L(P, L$-произвольные ЛДОЧП). В следуюшем разделе будет показано, что не все (левые или правые) идеалы кольца ЛДОЧП дивизорные.

Дивизорная пара идеалов $\{L|M| R\}$ называется тривиальной, если $\{L \mid=\langle M|$ (тогда $\mid R\}=|1\rangle)$ либо $\mid R\}=|M\rangle(\{L \mid=\langle 1|)$.

Для двух r.d.i. $\left.\left.\mid R_{1}\right\}, \mid R_{2}\right\}$ возьмем их пересечение в качестве их левого наименьшего обшего кратного (lLCM): $\left.\left.\left.\left.\operatorname{lLCM}\left(\mid R_{1}\right\}, \mid R_{2}\right\}\right):=\mid R_{1}\right\} \cap \mid R_{2}\right\}$. Тогда для $\left.\left.M \in \mid R_{1}\right\} \cap \mid R_{2}\right\}$ 
мы можем найти два соответствуюших l.d.i. (лемма 3): $\left\{L_{1}|M| R_{1}\right\},\left\{L_{2}|M| R_{2}\right\}$. Теперь возьмем все ЛДОЧП $L$ такие, что (9) выполнено для каждого $\left.\left.R \in \mid R_{1}\right\} \cap \mid R_{2}\right\}$. Правый идеал $\{L \mid$ таких операторов образует дивизорную пару идеалов с $\left.\left.\mid R\}=\mid R_{1}\right\} \cap \mid R_{2}\right\}$ для $M$, поскольку условия "а" и "б" в определении 3 выполняются автоматически и $\left\{L \mid \supset\left\{L_{1} \mid \cup\left\{L_{2} \mid\right.\right.\right.$, поэтому всякий оператор $R$ такой, что (9) выполнено для всех $L \in\left\{L \mid\right.$, образует дивизорную пару операторов со всеми $L \in\left\{L_{1} \mid\right.$ и $L \in\left\{L_{2} \mid\right.$, откуда $\left.\left.R \in \mid R_{1}\right\} \cap \mid R_{2}\right\}$ и условие "в" также выполняется. Как мы увидим в следуюшем разделе, построенный идеал $\left\{L \mid\right.$ в общем случае больше, чем множество всех сумм элементов $\left\{L_{1} \mid\right.$ и $\left\{L_{2} \mid\right.$. Очевидно, что этот идеал $\left\{L \mid\right.$ играет роль $\operatorname{lGCD}\left(\left\{L_{1} \mid,\left\{L_{2} \mid\right)\right.\right.$ во множестве всех 1.d.i (GCD - greatest common divisor). Определение $\left.\left.\mid R\}=\operatorname{rGCD}\left(\mid R_{1}\right\}, \mid R_{2}\right\}\right)$ формулируется аналогичным образом: возьмем теперь $\left\{L \mid=\operatorname{rLCM}\left(\left\{L_{1} \mid,\left\{L_{2} \mid\right):=\left\{L_{1} \mid \cap\left\{L_{2} \mid\right.\right.\right.\right.\right.$; тогда соответствуюший $\left.\left.\mid R\} \supset \mid R_{1}\right\} \cup \mid R_{2}\right\}$ определим, используя (9).

\section{4. КООР ДИНАТИЗАЦИЯ ДИВИЗОРНЫХ ИДЕАЛОВ}

Любое (некоммутативное в общем случае) кольцо $R$, удовлетворяюшее так называемому условию Оре (отсутствие делителей нуля и сушествование по крайней мере одного правого и одного левого общих кратных для любых двух ненулевых элементов), может быть вложено в тело (некоммутативный по умножению аналог поля), образованное формальными дробями (частными) $L^{-1} \circ M=\bar{M} \circ \bar{L}^{-1}, L, M, \bar{L}, \bar{M} \in R$ [14]. Возьмем $R=\mathbf{Q}(x, y)\left[D_{x}\right]$ и соответствующее тело частных $\mathbf{Q}\left(x, y, D_{x}\right)$. Мы можем образовать кольцо $\mathbf{Q}\left(x, y, D_{x}\right)\left[D_{y}\right]$ операторов типа $L=a_{0} D_{y}^{n}+a_{1} D_{y}^{n-1}+\cdots+a_{n}$, $a_{i}=\left(L_{i}\right)^{-1} \circ M_{i} \in \mathbf{Q}\left(x, y, D_{x}\right)$, если мы определим $D_{y}$-дифференширование для коэффициентов

$$
\frac{\partial\left(\left(L_{i}\right)^{-1} \circ M_{i}\right)}{\partial y}=\left(L_{i}\right)^{-1} \circ \frac{\partial\left(M_{i}\right)}{\partial y}-\left(L_{i}\right)^{-1} \circ L_{i}^{\prime} \circ\left(L_{i}\right)^{-1}
$$

$$
\frac{\partial\left(M_{i}\right)}{\partial y}=\frac{\partial\left(m_{0}(x, y) D_{x}^{k}+\cdots+m_{k}(x, y)\right)}{\partial y}=\frac{\partial m_{0}(x, y)}{\partial y} D_{x}^{k}+\cdots+\frac{\partial m_{k}(x, y)}{\partial y} .
$$

Элемент $L_{i}^{\prime} \in \mathbf{Q}(x, y)\left[D_{x}\right]$ определяется очевидным образом:

$$
D_{y} \circ L_{i}=L_{i} \circ D_{y}+L_{i}^{\prime} \Rightarrow L_{i}^{-1} \circ D_{y}=D_{y} \circ L_{i}^{-1}+L_{i}^{-1} \circ L_{i}^{\prime} \circ L_{i}^{-1} .
$$

Как объясняется в [10], основные факты теории Леви-Оре (именно наличие алгоритма Евклида, наибольших обших делителей, наименьших обших кратных и т.п.) верны также для операторов с коэффишиентами в дифференциальных телах. Любой правый или левый идеал вновь является главным, и вьполняются все основные теоремы теории факторизации ЛОДО.

Мы имеем естественные проекции

$$
\mathcal{P}_{x}: \mathbf{Q}(x, y)\left[D_{x}, D_{y}\right] \rightarrow \mathbf{Q}\left(x, y, D_{y}\right)\left[D_{x}\right]
$$

и

$$
\mathcal{P}_{y}: \mathbf{Q}(x, y)\left[D_{x}, D_{y}\right] \rightarrow \mathbf{Q}\left(x, y, D_{x}\right)\left[D_{y}\right]
$$


Для любого левого идеала $I \subset \mathbf{Q}(x, y)\left[D_{x}, D_{y}\right]$ имеются два главных левых идеала $\mathcal{P}_{x}(I)=\left|I_{x}\right\rangle, \mathcal{P}_{y}(I)=\left|I_{y}\right\rangle$. Порождаюшие их $I_{x}, I_{y}$ назовем координатами идеала $I$; $I_{x} \in \mathbf{Q}\left(x, y, D_{y}\right)\left[D_{x}\right], I_{y} \in \mathbf{Q}\left(x, y, D_{x}\right)\left[D_{y}\right]$. Нормализуем их условием $\operatorname{lcof}_{D_{x}}\left(I_{x}\right)=1$, $\operatorname{lcof}_{D_{y}}\left(I_{y}\right)=1$. Строго говоря, $\mathcal{P}_{x}(I)$ и $\mathcal{P}_{y}(I)$ не являются идеалами в соответствующих кольцах. Для того чтобы образовать нужные нам идеалы-проекции, необходимо умножать их элементы на всевозможные операторы $L^{-1}, L \in \mathbf{Q}(x, y)\left[D_{x}\right]$ (соответственно $\left.L \in \mathbf{Q}(x, y)\left[D_{y}\right]\right)$. В дальнейшем это всегда будет подразумеваться.

Прежде всего заметим, что если мы имеем пару $\{L|M| R\}$, то для координат выполняется условие

$$
\mathcal{P}_{x}(M)=L_{x} \circ R_{x}
$$

это дает эвристическое обоснование определений предыдушего раздела. Кроме того, как очевидно,

$$
\left.\left.\left.\left.\mathcal{P}_{x}\left(\operatorname{lLCM}\left(\mid R_{1}\right\}, \mid R_{2}\right\}\right)\right)=\operatorname{lLCM}\left(\mathcal{P}_{x}\left(\mid R_{1}\right\}\right), \mathcal{P}_{x}\left(\mid R_{2}\right\}\right)\right),
$$

а это, в свою очередь, приводит к

$$
\mathcal{P}_{x}\left(\operatorname { l G C D } \left(\left\{L_{1} \mid,\left\{L_{2} \mid\right)\right)=\operatorname{lGCD}\left(\mathcal { P } _ { x } \left(\left\{L_{1} \mid\right), \mathcal{P}_{x}\left(\left\{L_{2} \mid\right)\right)\right.\right.\right.\right.
$$

в силу (14). Аналогично

$$
\begin{aligned}
& \left.\left.\left.\left.\mathcal{P}_{x}\left(\operatorname{rGCD}\left(\mid R_{1}\right\}, \mid R_{2}\right\}\right)\right)=\operatorname{rGCD}\left(\mathcal{P}_{x}\left(\mid R_{1}\right\}\right), \mathcal{P}_{x}\left(\mid R_{2}\right\}\right)\right), \\
& \mathcal{P}_{x}\left(\operatorname { r L C M } \left(\left\{L_{1} \mid,\left\{L_{2} \mid\right)\right)=\operatorname{rLCM}\left(\mathcal { P } _ { x } \left(\left\{L_{1} \mid\right), \mathcal{P}_{x}\left(\left\{L_{2} \mid\right)\right) .\right.\right.\right.\right.
\end{aligned}
$$

Следуюшая лемма играет ключевую роль в дальнейших доказательствах.

Лемма 4. Eсли r.d.i. $\mid R\} \subset \mathbf{Q}(x, y)\left[D_{x}, D_{y}\right]$ содерәит два әлемента $A \circ P, B \circ P$ таких, что $A \in \mathbf{Q}(x, y)\left[D_{x}\right], B \in \mathbf{Q}(x, y)\left[D_{y}\right]$, mо $\left.P \in \mid R\right\}$.

Доказательство. Используя (10), получаем $A \circ P \circ \bar{X}=Q \circ \bar{Y}, B \circ P \circ \bar{X}=S \circ \bar{Y}$ для некоторых $S, Q$. В этом случае

$$
\begin{gathered}
P \circ \bar{X}=A^{-1} \circ Q \circ \bar{Y} \in \mathbf{Q}\left(x, y, D_{x}\right)\left[D_{y}\right], \\
B \circ P \circ \bar{X}=B \circ A^{-1} \circ Q \circ \bar{Y}=S \circ \bar{Y},
\end{gathered}
$$

т.е.

$$
B \circ A^{-1} \circ Q=S \in \mathbf{Q}(x, y)\left[D_{x}, D_{y}\right],
$$

$B \in \mathbf{Q}(x, y)\left[D_{x}\right]$. Покажем, что тогда $Q$ делится на $A: Q=A \circ \bar{Q}, \bar{Q} \in \mathbf{Q}(x, y)\left[D_{x}, D_{y}\right]$. Без потери общности предположим, что $\operatorname{lcof}(B)=1$ и формула (15) принимает вид

$$
\underbrace{\left(D_{y}^{m}+b_{1}(x, y) D_{y}^{m-1}+\cdots+b_{m}(x, y)\right)}_{B} \circ \underbrace{\left(C_{0} D_{y}^{n}+C_{1} D_{y}^{n-1}+\cdots+C_{n}\right)}_{A^{-1} \circ Q}=S
$$

с $C_{i} \in \mathbf{Q}\left(x, y, D_{y}\right)$. Старший коэффишиент (в $\left.\mathbf{Q}\left(x, y, D_{x}\right)\left[D_{y}\right]\right)$ левой части $(16)$ есть $C_{0}$, и поскольку $S \in \mathbf{Q}(x, y)\left[D_{x}, D_{y}\right]$, то $C_{0} \in \mathbf{Q}(x, y)\left[D_{x}\right]$. Следовательно, коэффициент $D_{y}^{m+n-1}$ в (16) будет $C_{1}+\partial C_{0} / \partial y+b_{1} C_{0} \in \mathbf{Q}(x, y)\left[D_{x}\right]$, т.е. $C_{1} \in \mathbf{Q}(x, y)\left[D_{x}\right]$. Используя индукцию, получаем $C_{i} \in \mathbf{Q}(x, y)\left[D_{x}\right]$, откуда $A^{-1} \circ Q=\bar{Q} \in \mathbf{Q}(x, y)\left[D_{x}, D_{y}\right]$. Это дает нам возможность сократить $A$ в равенстве $A \circ \bar{X}=Q \circ \bar{Y}$, получив $\bar{X}=\bar{Q} \circ \bar{Y}$. Отсюда имеем $P \in \mid R\}$, поскольку $P \circ \bar{X}=(P \circ \bar{Q}) \circ \bar{Y}$ в (10). 
Следствие 1. Если r.d.i. $\mid R\}$ содержит два әлемента $A, B$ и $A \in \mathbf{Q}(x, y)\left[D_{x}\right]$, $B \in \mathbf{Q}(x, y)\left[D_{y}\right]$, то идеал $\left.\mid R\right\}$ тривиален, т.е. $\left.\mid R\right\}=|1\rangle=\mathbf{Q}(x, y)\left[D_{x}, D_{y}\right]$.

Это следствие объясняет, почему r.d.i. следует считать идеалами "коразмерности $n-1$ ": если мы возьмем левый идеал “коразмерности $n-2$ ", порожденный $D_{x}^{n}, D_{y}^{m}$ (решениями соответствуюшей системы $D_{x}^{n} f=0, D_{y}^{m} f=0$ являются функции $n-2$ переменных, параметризованные несколькими константами, а не функции $n-1$ переменной), то этот идеал содержится лишь в тривиальном r.d.i. $\mid 1\}$. Множество дивизорных идеалов несколько больше множества главных идеалов (идеалы "коразмерности $n-1$ ", являются решениями соответствуюшей системы из одного уравнения и параметризуются функциями $n-1$ переменной).

Tеорема 4. Два r.d.i. $\left.\left.\mid R_{1}\right\}, \mid R_{2}\right\}$ совпадают, если и только если их координаты совпадают, т.е. $\left.\left.\left.\left.\mathcal{P}_{x}\left(\mid R_{1}\right\}\right)=\mathcal{P}_{x}\left(\mid R_{2}\right\}\right), \mathcal{P}_{y}\left(\mid R_{1}\right\}\right)=\mathcal{P}_{y}\left(\mid R_{2}\right\}\right)$.

ДокАЗАтельСтво. Выберем $\left.R \in \mid R_{1}\right\}$. Поскольку $\left.\left.\mathcal{P}_{x}\left(\mid R_{1}\right\}\right)=\mathcal{P}_{x}\left(\mid R_{2}\right\}\right)$, мы можем найти такой $\left.\bar{R} \in \mid R_{2}\right\}$, что $C$ 。 $R=\bar{R}, C=\left(C_{1}\right)^{-1} C_{2} \in \mathbf{Q}\left(x, y, D_{y}\right)$. Умножая на $C_{1}$, получаем $\left.C_{2} \circ R=C_{1} \circ \bar{R}=\widetilde{R} \in \mid R_{2}\right\}$. Следовательно, для некоторого $C_{2} \in \mathbf{Q}(x, y)\left[D_{y}\right]$ имеем $\left.C_{2} \circ R \in \mid R_{2}\right\}$. Аналогичные рассмотрения для $\mathcal{P}_{y}$ дают $\left.K_{2} \circ R \in \mid R_{2}\right\}$ для некоторого $K_{2} \in \mathbf{Q}(x, y)\left[D_{x}\right]$, откуда $\left.R \in \mid R_{2}\right\}$ (лемма 4 ), т.е. $\left.\left.\mid R_{1}\right\} \subset \mid R_{2}\right\}$. Аналогично получаем $\left.\left.\mid R_{2}\right\} \subset \mid R_{1}\right\}$.

ЗАмЕчАниЕ. Это утверждение неверно для произвольных идеалов: идеал, порожденный $D_{x}, D_{y}$, имеет те же проекции, что и тривиальньй идеал $|1\rangle$.

\section{5. МОДУЛЯРНОСТЬ РЕШЕТКИ ДИВИЗОРНЫХ ИДЕАЛОВ}

Теперь мы можем доказать все необходимые свойства обобщенной факторизации на основе определений предыдуших разделов. Для этого мы используем обший подход к доказательству теорем типа Жордана-Гельдера в рамках теории частично упорядоченных множеств специального типа - решеток. Введем очевидное упорядочение на множестве (левых) идеалов: $I_{1} \leqslant I_{2}$, если $I_{1} \supset I_{2}$; назовем в этом случае $I_{1}$ делителем (дивизором) $I_{2}$. Частично упорядоченное множество $\mathcal{M}$ идеалов в упомянутых во введении случаях наличия "хорошей” теории факторизации обладает следуюшими важными свойствами:

А. Для любых двух элементов $A, B \in \mathcal{M}$ сушествует единственный элемент $C=$ $\sup (A, B)$, т.е. такой элемент, что $C \geqslant A, C \geqslant B$ и $\forall X \in \mathcal{M}(X \geqslant A, X \geqslant B) \Rightarrow$ $X \geqslant C$. Аналогично существует единственный элемент $D=\inf (A, B)$ такой, что $D \leqslant A, D \leqslant B, \forall X \in \mathcal{M}(X \leqslant A, X \leqslant B) \Rightarrow X \leqslant D$. Такие частично упорядоченные множества называются решетками. Элементы $\sup (A, B)$ и $\inf (A, B)$ соответствуют наименьшему обшему кратному и наибольшему обшему делителю в случае числовых, полиномиальных колец и ЛОДО. В наших случаях решетка всегда имеет нуль, т.е. элемент $0 \in \mathcal{M}$ такой, что $\forall X \in \mathcal{M} X \geqslant 0$. Для краткости (и следуя установившейся в теории решеток традиции) $\sup (A, B)$ в дальнейшем мы будем обозначать как $A+B$, а $\inf (A, B)-$ как $A \cdot B$. 
Б. Для любых трех элементов $A, B, C \in \mathcal{M}$ выполнено следуюшее модулярное тождество:

$$
(A \cdot C+B) \cdot C=A \cdot C+B \cdot C .
$$

Эта слабая форма дистрибутивности была открыта Дедекиндом. Теория модулярных решеток, т.е. частично упорядоченных множеств, в которых выполнены оба вышеприведенных свойства (такие решетки назывались ранее “дедекиндовыми структурами"), содержит все необходимые для нас результаты. Именно данных двух простых свойств достаточно для доказательства следуюших теорем [15-17]:

Теорема 5 (теорема Жордана-Гельдера-Дедекинда). Любые две конечные максимальные чепочки

$$
\begin{gathered}
L>L_{1}>\cdots>L_{k}>0, \\
L>M_{1}>\cdots>M_{r}>0
\end{gathered}
$$

для заданного әлемента $L \in \mathcal{M}$ модулярной решетки имеют равную длину: $k=r$.

Tеорема 6 (Курош-Оре). Если $L=L_{1}+L_{2}+\cdots+L_{p}=M_{1}+M_{2}+\cdots+M_{r}$ два несократимых sup-представления $L \in \mathcal{M}$, то $p=r$ и для любого $L_{i}$ можно найти $M_{j}$ такой, что $L=L_{1}+\cdots+L_{i-1}+M_{j}+L_{i+1}+\cdots+L_{p}$.

Напомним, что sup-представление

$$
L=L_{1}+L_{2}+\cdots+L_{k}
$$

называется несократимым, если для любого $i$ имеем $\left(L_{1}+\cdots+L_{i-1}+L_{i+1}+\cdots+L_{k}\right) \neq$ $L$ и каждый $L_{i}$ не может быть нетривиально sup-разложен: $L_{i} \neq A_{i}+B_{i}$ с $A_{i} \neq L_{i}$, $B_{i} \neq L_{i}$. Sup-представление (19) называется прямой суммой, если множество элементов $\left\{L_{i}\right\}$ независимо, т.е. для любого $i$ имеем $\left(L_{1}+\cdots+L_{i-1}+L_{i+1}+\cdots+L_{k}\right) \cdot L_{i}=0$ и каждый $L_{i}$ не может быть представлен как сумма двух независимых элементов. Прямые суммы обозначаются следуюшим образом: $L=L_{1} \oplus L_{2} \oplus \cdots \oplus L_{k}$. Элемент $A \in \mathcal{M}$ называется неразложимым, если $A \neq B \oplus C, B \neq 0, C \neq 0$.

Теорема 7 (Оре). Пусть әлемент L модулярной решетки имеет конечные максимальные чепочки (18) и $L=L_{1} \oplus \cdots \oplus L_{k}=M_{1} \oplus \cdots \oplus M_{r}$ с неразложимыми $L_{i}, M_{j}$. Тогда $k=r u \forall L_{i}$ найдется такой $M_{j}$, ито $L=L_{1} \oplus \cdots \oplus L_{i-1} \oplus M_{j} \oplus$ $L_{i+1} \oplus \cdots \oplus L_{p}$.

Назовем число $l(L):=k+1$ длиной элемента $L \in \mathcal{M}$, если $L$ имеет конечную максимальную цепочку (18) (длины $k+1)$. Положим $l(0)=0$. Длина ЛОДО равна числу неприводимых множителей в разложении (7).

Теорема 8. Если все әлементы модулярной решетки $\mathcal{M}$ имеют конечную длину, mо $l(A+B)+l(A \cdot B)=l(A)+l(B)$.

Тем самым теория модулярных решеток заключает в себе многие факты теории групп (и их представлений), коммутативных и некоммутативных колец; в частности, теоремы 5-8 включают как частные случаи многие результаты теории Леви-Оре факторизации ЛОДО. 
Докажем для полноты изложения, что частично упорядоченное множество (левых) идеалов любого (некоммутативного) кольца представляет собой модулярную решетку $[15,16]$. Прежде всего заметим, что $\sup (A, B)=A+B$ соответствует пересечению идеалов $A, B ; \inf (A, B)=A \cdot B$ соответствует идеалу, порожденному суммами $a+b, a \in A, b \in B$. Если $x \in A \cdot C+B \cdot C$ (правая часть (17)), то $x \in C, x=a+b$, $a \in A, a \in C, b \in B, b \in C$. Очевидно, что $a+b \in C, a+b \in A \cdot C+B$, поэтому $A \cdot C+B \cdot C \subset(A \cdot C+B) \cdot C$. Обратно, если $x \in(A \cdot C+B) \cdot C$, то $x \in C, x=a+b$, $a \in A, a \in C, b \in B \Rightarrow b=x-a \in C, b \in B \cdot C$ и $x \in A \cdot C+B \cdot C$, что и доказывает вложение $(A \cdot C+B) \cdot C \subset A \cdot C+B \cdot C$.

В рамках теории модулярных решеток также имеется возможность определить понятие "сходства" промежутков максимальных цепочек в теореме 5, что соответствует сходству операторов в различных факторизациях ЛОДО (7) или изоморфизму фактор-групп (фактор-модулей) в цепочках Жордана-Гельдера.

К сожалению, невозможно использовать свойство модулярности множества всех (левых или правых) идеалов в $\mathbf{Q}(x, y)\left[D_{x}, D_{y}\right]$, доказанное выше: как мы видели, цепочки идеалов в этом случае обычно имеют неограниченную длину, поэтому теоремы $5-8$ неприменимы.

Tеорема 9. Решетка r.d.i. (1.d.i.) модулярна.

ДокАЗАТЕЛЬСТво. Поскольку модулярное тождество (17) выполнено для проекций $\mathcal{P}_{x}, \mathcal{P}_{y}$ в силу модулярности решетки (главных) левых идеалов в $\mathbf{Q}\left(x, y, D_{x}\right)\left[D_{y}\right]$, $\mathbf{Q}\left(x, y, D_{y}\right)\left[D_{x}\right]$ и $\mathrm{GCD}, \mathrm{LCM}$ сохраняются при проекции, можно заключить, что $\mathcal{P}_{x}((A \cdot C+B) \cdot C)=\mathcal{P}_{x}(A \cdot C+B \cdot C), \mathcal{P}_{y}((A \cdot C+B) \cdot C)=\mathcal{P}_{y}(A \cdot C+B \cdot C)$, откуда имеем $(A \cdot C+B) \cdot C=A \cdot C+B \cdot C$.

СлЕДСТВИЕ 2. Для всякого r.d.i. $\mid R\}$ длина $k+1$ чепочки r.d.i. $\mid R\}>$ $\left.\left.\mid R_{1}\right\}>\cdots>\mid R_{k}\right\}>0$ ограничена: $\left.\left.k+1 \leqslant \operatorname{ord}_{D_{x}}(\mid R\}_{x}\right)+\operatorname{ord}_{D_{y}}(\mid R\}_{y}\right)$.

ДокАЗАТЕЛЬСТво. Поскольку $\left.\left.\mathcal{P}_{x}\left(\mid R_{i}\right\}\right), \mathcal{P}_{y}\left(\mid R_{i}\right\}\right)$ дают цепочки дивизоров для $\left.\left.\mathcal{P}_{x}(\mid R\}\right), \mathcal{P}_{y}(\mid R\}\right)$ в $\mathbf{Q}\left(x, y, D_{y}\right)\left[D_{x}\right]$ (соответственно $\left.\mathbf{Q}\left(x, y, D_{x}\right)\left[D_{y}\right]\right)$, соседние элементы $\mathcal{P}_{x}$-спроецированной цепочки могут отличаться лишь в $\left.\operatorname{ord}_{D_{x}}(\mid R\}_{x}\right)$-местах (соответственно в $\left.\operatorname{ord}_{D_{y}}(\mid R\}_{y}\right)$-местах).

Аналогичный результат верен для (прямых) sup-cyмм r.d.i.

Таким образом, теоремы 5-8 могут быть применены к построенной нами решетке r.d.i. (l.d.i.) заданного ЛДОЧП.

УТВЕРЖДЕНИЕ 1. Любой ЛДОЧП $R$ первого порядка неприводим, т.е. не имеет нетривиальных дивизорных пар идеалов.

ДокАЗАТЕЛЬСтво. Пусть $R=r_{1}(x, y) D_{x}+r_{2}(x, y) D_{y}+r_{3}(x, y)$, но тем не менее имеются некоторые r.d.i. $\left.\left.\left.\mid R_{1}\right\}, \quad \mid R\right\}>\mid R_{1}\right\}>0$. С необходимостью имеем $\left.\left.\operatorname{ord}_{D_{x}}\left(\mid R_{1}\right\}_{x}\right)=1, \operatorname{ord}_{D_{y}}\left(\mid R_{1}\right\}_{y}\right)=0$ (с точностью до перестановки $\left.x \leftrightarrow y\right)$, поэтому существуют

$$
\begin{gathered}
\left.R_{1,1}=C_{0} \circ D_{x}+C_{1} \in \mid R_{1}\right\}, \\
\left.R_{1,2}=K_{1} \in \mid R_{1}\right\}, \quad C_{i} \in \mathbf{Q}(x, y)\left[D_{y}\right], \quad K_{1} \in \mathbf{Q}(x, y)\left[D_{x}\right] .
\end{gathered}
$$


Так как $\left.\mid R\} \subset \mid R_{1}\right\}$, получаем

$$
\left.R_{1,3}=R_{1,1}-\frac{C_{0}}{r_{1}(x, y)} R=K_{2} \in \mid R_{1}\right\}, \quad K_{2} \in \mathbf{Q}(x, y)\left[D_{y}\right] .
$$

В силу следствия 1 леммы 4 имеем $\left.\mid R_{1}\right\}=0=\mathbf{Q}(x, y)\left[D_{x}, D_{y}\right]$.

УТВЕРЖДЕНИЕ 2. Любой ЛОДО $M \in \mathbf{Q}(x)\left[D_{x}\right]$, неприводимый в этом кольце, неприводим как әлемент $\mathbf{Q}(x, y)\left[D_{x}, D_{y}\right]$.

ДоКАЗАТЕЛЬСТво. Предположим, что мы имеем нетривиальную дивизорную пару идеалов $\{L|M| R\}$; тогда для некоторого $L \in\{L|, R \in| R\}$ выполнено условие (10). Поскольку $M \in\{L|, M \in| R\}$, можно предположить (вычитая подходящее кратное оператора $M)$, что $\operatorname{ord}_{D_{x}}(L)=\operatorname{ord}\left(\mathcal{P}_{x}(L)\right)<\operatorname{ord}_{D_{x}}(M)=m, \operatorname{ord}_{D_{x}}(R)<m$. Образуя тело частных $\mathbf{Q}\left(x, y, D_{x}, D_{y}\right)$, мы можем в этом теле написать

$$
X=Y \circ Q \circ L^{-1} \Rightarrow X \circ M=Y \circ Q \circ L^{-1} \circ M=Y \circ R \Rightarrow Q \circ L^{-1} \circ M=R .
$$

Если мы найдем

$$
\operatorname{lLCM}\left(\mathcal{P}_{x}(Q), \mathcal{P}_{x}(L)\right)=Z=\bar{Q} \circ L=\bar{L} \circ Q_{x} \in \mathbf{Q}\left(x, y, D_{y}\right)\left[D_{x}\right],
$$

то $\operatorname{ord}_{D_{x}}(\bar{L}) \leqslant \operatorname{ord}_{D_{x}}(L)<m$. Для некоторого $C \in \mathbf{Q}(x, y)\left[D_{y}\right]$ будет верно

$$
\begin{aligned}
C \circ \bar{Q} & =\widetilde{Q} \in \mathbf{Q}(x, y)\left[D_{x}, D_{y}\right], \\
C \circ \bar{L} & =\tilde{L} \in \mathbf{Q}(x, y)\left[D_{x}, D_{y}\right], \quad \operatorname{ord}_{D_{x}}(\tilde{L})=\operatorname{ord}_{D_{x}}(\bar{L})<m .
\end{aligned}
$$

Следовательно, $\tilde{L} \circ Q \circ L^{-1} \circ M=\tilde{L} \circ R \Leftrightarrow \widetilde{Q} \circ L \circ L^{-1} \circ M=\widetilde{Q} \circ M=\tilde{L} \circ R$. Возьмем

$$
\begin{aligned}
\operatorname{lcof}_{D_{y}}(\tilde{L} \circ R): & =\operatorname{lcof}\left(\mathcal{P}_{y}(\tilde{L} \circ R)\right)=\operatorname{lcof}_{D_{y}}(\tilde{L}) \cdot \operatorname{lcof}_{D_{y}}(R)= \\
& =C_{\tilde{L}} \circ C_{R} \in \mathbf{Q}(x, y)\left[D_{x}\right], \quad \operatorname{ord}\left(C_{\tilde{L}}\right)<m, \quad \operatorname{ord}\left(C_{R}\right)<m, \\
\operatorname{lcof}_{D_{y}}(\widetilde{Q} \circ M) & =\operatorname{lcof}_{D_{y}}(\widetilde{Q}) \circ M=C_{\widetilde{Q}} \circ M, \quad \operatorname{ord}(M)=m .
\end{aligned}
$$

Далее получаем $C_{\tilde{L}} \circ C_{R}=C_{\widetilde{Q}} \circ M$ в $\mathbf{Q}(x, y)\left[D_{x}\right]$, что противоречит неприводимости $M$ и тому факту, что $\operatorname{ord}(M)=m$, в то время как порядки факторов в левой части меньше $m$.

Теперь мы можем объяснить появление примера Ландау (5): в силу утверждения 1 цепочка $\mid Q \circ Q \circ P\}>\mid Q \circ P\}>\mid P\}>0$ максимальна, поэтому единственное место, где может появиться обобшенный дивизорный идеал - интервал между $\mid R \circ Q\}$ и $\mid Q\}$ в $\mid R \circ Q\}>\mid Q\}>0$. Именно мы можем вставить здесь $I=\operatorname{lLCM}(\mid Q\}, \mid P\})$. Проекция $\mathcal{P}_{x}(I)$ порождена оператором второго порядка (относительно $\left.D_{x}\right) L_{31}=\left(x^{2} D_{y}^{2}+\right.$ $\left.x D_{x} D_{y}-(x+1) D_{y}-D_{x}\right) \circ Q$, в то время как $\mathcal{P}_{x}(R \circ Q)$ - оператор третьего порядка. Поэтому оператор $R$ приводим в нашем смысле (и приводим в $\mathbf{Q}\left(x, y, D_{y}\right)\left[D_{x}\right]$ ), он имеет нетривиальный “обобшенный общий (правый) делитель" с $x^{2} D_{y}^{2}+x D_{x} D_{y}-(x+1) D_{y}-D_{x}$ (определение 2). 


\section{6. ОБОБШЕННАЯ ФАКТОРИЗУЕМОСТЬ ОПЕРАТОРОВ ЛАПЛАСА}

В этом разделе мы докажем теоремы 2 и 3 (упомянутые во введении). Предположим, что оператор

$$
L=D_{x} \circ D_{y}-a(x, y) D_{x}-b(x, y) D_{y}-c(x, y)
$$

имеет нетривиальный r.d.i. $\mid R\}$, т.е. мы имеем некоторый нетривиальный ЛДОЧП $P \in \mid R\}, P \notin|L\rangle$. Всегда можно предположить (по модулю $L$ ), что $P$ не имеет слагаемых со смешанными производными, т.е.

$$
P=\sum_{i=1}^{n} p_{i}(x, y) D_{x}^{i}+\sum_{j=1}^{m} q_{j}(x, y) D_{y}^{j}+c_{0}(x, y)
$$

$p_{n} \neq 0, q_{m} \neq 0$.

Лемма 5. Если в формуле (21) $n>0, m>0$, то $\mid R\}$ тривиален.

ДокаЗАТЕльство. Деля $D_{x} P$ на $L$ с остатком, получаем оператор со старшими членами $p_{n} D_{x}^{n+1}, q_{m} b D_{y}^{m}$. Второй из них может быть приведен к нулю по модулю $P$. Через некоторое количество таких шагов мы придем к элементу идеала $\mid R\}$, имеюшему лишь $D_{x}$-производные. Аналогично мы найдем элемент $\left.\mid R\right\}$ с чистыми $D_{y}$-производными. Из следствия 1 получаем, что $\mid R\}=|1\rangle$.

Итак, нетривиальный r.d.i. для $L$ содержит в точности один минимальный элемент $P$ либо с чистыми $D_{x^{-}}$, либо с чистыми $D_{y}$-производными, и система из двух уравнений $L=0, P=0$ находится в инволюции (совместна): $D_{y} P$ (соответственно $D_{x} P$ ) приводится к нулю по модулю $L$. Следуюший результат был доказан в [18].

ЛЕмма 6. Если уравнение второго порядка (20) находится в инволюиии с линейнымм уравнением порядка п с чистыми $D_{x}$ - либо $D_{y}$-производными, то чепочка преобразований Лапласа для (20) конечна в одну из сторон.

Теорема 2 является простым следствием этих двух лемм. Теорема 3 вытекает из того факта, что для двух r.d.i. $\left.\mid R_{1}\right\}=\left|L, P_{1}\left(x, y, D_{x}\right)\right\rangle$ и $\left.\mid R_{2}\right\}=\left|L, P_{2}\left(x, y, D_{y}\right)\right\rangle$ операторы $D_{y} P_{1}$ и $D_{x} P_{2}$ приводятся к нулю по модулю $L$, поэтому $\left.\left.\mid R_{1}\right\} \cap \mid R_{2}\right\}=|L\rangle$. Это также отражает тот факт (см., например, [5]), что пространство решений уравнения $L u=0$ в этом случае есть (не прямая) сумма двух подпространств (пространств решений для идеалов $\left.\mid R_{1}\right\}$ и $\left.\mid R_{2}\right\}$, оба подпространства параметризуются функциями одного соответствуюшего переменного):

$$
u=u_{1}+u_{2}, \quad u_{1}=\sum_{i=0}^{n-1} \phi_{i}(x, y) D_{y}^{i}(Y(y)), \quad u_{2}=\sum_{j=0}^{m-1} \psi_{j}(x, y) D_{x}^{i}(X(x))
$$

с произвольными $X(x), Y(y)$. Тем самым подобные r.d.i. имеют сходство с операторами первого порядка. 


\section{7. ЗАКЛЮЧЕНИЕ}

Полученные результаты позволяют выдвинуть следуюшую гипотезу.

ГипотеЗА. Скалярное нелинейное уравнение с частнылми производными с несколькими независимыми и одной зависимой переменньми является "интегрируемым по Дарбу" тогда и только тогда, когда соответствующий линеаризованный оператор может быть представлен как левое наименьшее общее кратное нескольких r.d.i., каждый из которых имеет "порядок 1 ", т.е. имеет пространство решений, параметризуемое одной функиией $n-1$ переменного.

Представляет интерес построить алгоритм разложения заданного ЛДОЧП в ILCM некоторого количества r.d.i. Следует отметить, что процедура, связанная с преобразованием Лапласа, не алгоритмична: нет возможности оценить количество необходимых для этого преобразований Лапласа. С другой стороны, результаты Дарбу [5, гл. 2] предоставляют в явной форме операторы Лапласа с цепочкой преобразований Лапласа, конечной в обоих направлениях, что может обеспечить необходимую оценку.

Кроме связи с интегрируемостью по Дарбу, представление ЛДОЧП в виде $\mathrm{lLCM}$ правых дивизорных идеалов имеет очевидное применение к упрощению решения переопределенных систем ЛДОЧП:

$$
\left\{\begin{array}{l}
L_{1} f=0, \\
L_{2} f=0, \\
\cdots \\
L_{k} f=0 .
\end{array}\right.
$$

Предположим, что $\left|L_{1}\right\rangle=R_{1}+\cdots+R_{p}$ для некоторых r.d.i. $R_{i}$. Тогда, поскольку каждый $R_{i}$ конечно порожден: $R_{i}=\left|L_{i 1}, \ldots, L_{i s_{i}}\right\rangle$, мы можем разложить $(22)$ в объединение "более простых" систем:

$$
\left\{\begin{array} { l } 
{ L _ { 1 1 } f = 0 , } \\
{ \ldots } \\
{ L _ { 1 s _ { 1 } } f = 0 , } \\
{ L _ { 2 } f = 0 , } \\
{ \cdots } \\
{ L _ { k } f = 0 , }
\end{array} \quad \ldots \quad \left\{\begin{array}{l}
L_{p 1} f=0 \\
\cdots \\
L_{p s_{p}} f=0 \\
L_{2} f=0, \\
\cdots \\
L_{k} f=0 .
\end{array}\right.\right.
$$

Обобшение понятия дивизорного идеала для систем ЛУЧП или одного ЛУЧП с несколькими зависимыми переменными (неизвестными функциями) пока неизвестны.

Возможные приложения предлагаемой техники дивизорных идеалов в теории солитонов (например, к биспектральным операторам) будут рассмотрены в других публикациях.

Благодарности. Данная работа получила финансовую поддержку Р $\Phi И$, грант № 98-01-00426, и Президентского гранта поддержки молодых докторов наук № $96-15-96834$. 


\section{Список литературы}

[1] G. Darboux. Ann. Ecole Normale Sup. 1870. V. VII. P. 163-173.

[2] I. M. Anderson, N. Kamran. Duke Math. J. 1997. V. 87. № 2. P. 265-319.

[3] M. Juras. Generalized Laplace invariants and classical integration methods for second order scalar hyperbolic partial differential equations in the plane. In: Proc. of the 6th Int. Conf. Brno, Czech Republik, Aug. 28-Sept. 1, 1995. Ed. J. Janyska. Brno: Masaryk University Press, 1996. P. 275-284.

[4] А. В. Жибер, В. В. Соколов, С. Я. Стариев. Докл. РАН. 1995. Т. 343. № 6. С. 746-748; V. V. Sokolov, A. V. Zhiber. Phys. Lett. A. 1995. V. 208. P. 303-308.

[5] G. Darboux. Leçons sur la théorie générale des surfaces et les applications géométriques du calcul infinitésimal. V.1-4. Paris: Gautier-Villar, 1887-1896.

[6] E. Landau. J. Reine Angew. Math. 1901/1902. V. 124. P. 115-120.

[7] S. P. Tsarev. An algorithm for complete enumeration of all factorizations of a linear ordinary differential operator. In: Proc. of the 1996 Int. Symp. on Symbolic and Algebraic Computation, ISSAC'96, Zuerich, Switzerland, July 24-26, 1996. Ed. Y. N. Lakshman. New York: ACM Press, 1996. P. 226-231.

[8] A. Loewy. Math. Ann. 1903. V. 56. P. 549-584.

[9] A. Loewy. Math. Ann. 1906. V. 62. P. 89-117.

[10] O. Ore. Ann. Math. 1933. V. 34. P. 480-508.

[11] E. Beke. Math. Ann. 1894. V. 45. P. 278-300.

[12] H. Blumberg. Über algebraische Eigenschaften von linearen homogenen Differentialausdrücken. Diss. Göttingen: Univ. Göttingen, 1912.

[13] К. Айерленд, М. Роузен. Классическое введение в современную теорию чисел. М.: Мир, 1987.

[14] O. Ore. Ann. Math. 1931. V. 32. P. 463-477.

[15] Г. Биржгоф. Теория решеток. М.: Мир, 1984.

[16] G. Grätzer. General lattice theory. Berlin: Akademie-Verlag, 1978.

[17] N. Jacobson. Lectures in abstract algebra. V.1. Basic Concepts. Toronto, New York, London: D. Van Nostrand Co., Inc, 1951.

[18] E. Goursat. Am. J. Math. 1896. V. 18. P. 347-385. 